\title{
Context-Aware Session and Network Control in Future Internet
}

\author{
Augusto Neto, Susana Sargento, Filipe Cabral Pinto, Evariste Logota
}

\begin{abstract}
With the rapidly increasing interest of citizens and businesses in multimedia group services, personalization, contextawareness and seamless mobility, the communication can no longer be limited to high speed network or devices. In future scenarios, context-aware services and multicasting will together drive a new trend, since context-awareness can exploit situations in which users share the same interests and request similar services, increasing the effectiveness of session and network control. However, context-aware networks impose several challenges: any change to context, such as, location, mobility, velocity, preferences, presence, etc., can change the overall services and network environments, requiring completely restructuring of the network and multicast sessions in a very dynamic way. This dynamicity imposes scalability problems in current networks.

In this paper, we present an initial future Internet architecture for context-aware network and service control, which enables the support of context-aware users and services, seamless reacting to the context changes with minimal impacts to the services and multicast control. For this purpose, we provide a network that is able to interact with context-based services, choose and change the session and network characteristics based on the context, both for the environment, user, network, and sessions. To address the dynamicity of the network, we provide the support of multicast abstract transport trees able to abstract the network changes to the running sessions transport, and limiting the impact of all the context dynamics in the user sessions.
\end{abstract}

Index Terms - Future Internet, Network Resource Provisioning, Context-Awareness, multicast and Mobility.

\section{INTRODUCTION}

$\mathrm{T}$ oday's Internet has been experiencing explosive demands for new types of multimedia sessions, in addition to current famous IPTV (web), e-learning, e-radio and video/audio conferencing. There is a special interest from mobile network providers in personalized and immersive (3D) services. However, the current Internet cannot efficiently support the strong requirements of such sessions, mainly due to the poor transport scheme, absence of wide multicast support, mobility and context un-awareness, as well as scalability concerns. Attempts to overcome, or alleviate, the shortcomings of currently Internet include the deployment of some of the functions on top of appropriate layers, such as is the case of resource provisioning, multicast (through overlays) and mobility. In spite of not

This work was supported in part by the FP7 C-CAST project.

A. Neto, S. Sargento and E. Logota are with the Telecommunications Institute, University of Aveiro, 3810-193, Aveiro, Portugal (e-mail: augusto@av.it.pt, susana@ua.pt,logota@av.it.pt).

F. Pinto is with Portugal Telecom Inovação, Aveiro, Portugal (e-mail: filipe-cpinto@ptinovacao.pt). requiring significant changes in network elements, such alternatives add complexity to the system and drastically endanger network performance. The introduction of contextawareness much complicates all the process: the possibility to recognize and react to context changes, being context related to users, sessions, network or the environment, requires the support of a very flexible session and network support system. The change in the context, e.g. requiring the sessions to shut down the voice system just keeping video, due to noise around, or to change specific users to different access networks to support a large event, requires the support of changes, both in the session and network sides, in a seamless and scalable way. In the future, more environment information will be included, resulting in smart spaces providing the specific context of shopping halls, airports, office, home etc., and will introduce large dynamicity to the user, session and network, in order to cope with the context changes. Thus, it is evident re-designing current Internet infrastructure to move towards a future approach.

The future Internet is envisioned to support a wide range of new multimedia services, aiming at allowing their access by fixed or mobile users ubiquitously. Therefore, the future wireless and wireline networks must be designed to be readily adaptable to current and future needs, at acceptable cost. In this sense, the FP7 C-CAST [1] project aims at evolving mobile multimedia multicasting to exploit the increasing integration of mobile devices with our everyday physical world and environment, providing an end-to-end context-aware communication framework specifically for intelligent multicast-broadcast services. One of its objectives is to define a framework to collect sensor data, distribute context information and manage efficiently context aware multiparty sessions and multicast transport.

The objective of this paper is to present an initial future Internet architecture, as part of the C-Cast architecture, for context-aware network and service control, which enables the support of context-aware users and services, seamless reacting to the context changes with minimal impacts to the services and multicast control. To that, this network contains mechanisms to support context acquisition and delivery, content matching according to the context, dynamic multi-party session control mechanism, intelligent decision of the best connections to the services and users according to the context information, and dynamic resource control and provisioning. To support all context changes with minimal impact and interference in the sessions and users, an abstract multiparty transport concept is devised, to create an abstract data path which is composed by the 
several sub transport structures, for further merging with multiple incoming multiparty content sessions. Abstract transport allows an end-to-end view in terms of connectivity, and will decouple all network changes and updates from the session transport.

This paper is organized as follows. Section II presents scenarios and challenges of context-aware networks. Section III briefly describes the initial architecture for context casting, and section IV details the session establishment and management parts. Finally, section V depicts the interactions between the elements in a future envisioned scenario, and section VI presents the final conclusions and future work.

\section{ENVIRONMENT SENSING AND CONTEXT-AWARENESS: SCENARIOS AND CHALLENGES}

The matching of personalized sessions can be influenced by several types of context, thus, allowing users access sessions based on their indoor location, preferences, profile and capabilities [2][3][4][5]. For instance, it should be desirable a mobile phone ringing mode to automatically switch to silence or vibration modes, once it appears to be in a classroom or at a meeting. In location-based advertisements, mobile users can receive asynchronous messages when their context satisfies predetermined conditions. In hospitals, keep track of patients who need urgent care, and detect doctors/nurses able to arrive rapidly are allowed. In tourism, announce museums and exhibitions close to the user, guide the visitors through halls, provide them explanations based on their locations and language. In emergency services which include gas explosions, aircraft crashes, earthquakes and train accidents where there is a major requirement to identify the presence of human beings within the disaster zones.

Today, there is a consensus that, to add a new flavour to the futuristic network convergence where different communication behaviours such as user-initiated communication mediated by the network, and situation-oriented and network-initiated communication mediated by the environment will become possible, the smart space needs to become integral part of the global group communications. A smart space [6][7][8] is any physical place with embedded services, thus bringing together the real and virtual worlds. It could be a well-defined enclosed area such as a meeting room or school or a well-defined open area such as a city square or national park. Typically a smart space comprises numerous heterogeneous sensors, smart devices and context information sinks, along with data servers with relevant (local public/environment) information which interact with each other to provide enriched services and hence facilitate user activities seamlessly.

Smart spaces are categorized as 1) user's personal space comprising of device and user context and 2) everything in the environment or user surroundings is considered as environment space. C-CAST will extend the concepts of smart spaces in the heterogeneous networking environment to provide the ubiquitous computing, and to the support of dynamic group management and personalization.
With the aim to efficiently support the scenarios described above, two set of functionalities must be deployed. In order to make environmental contexts available to eventually allow applications in context-aware behaviours, the following requirements must be addressed: (i) Obtain basic environmental context information from sensors and networks; (ii) Provide the information in an interpretable manner; (iii) Provide the necessary delivery service to make the information available; (iv) for groups of users according to context information. After that, it must be provided adaptive networking services to multiparty applications, with context-aware facilities to optimally use available networking resources and capabilities to best meet the varying application and user needs. The requirements include: $(i)$ Providing a generic (application-independent, networkindependent, content-independent and QoS-aware) networking service to allow applications to establish multiparty communications sessions with dynamic management of the joining and leaving of the session participants; (ii) Developing the necessary session and networking facilities to adapt, during the session, to needs and changes in session participants, user communications modes, user mobility, network availability, and network capabilities; and (iii) developing the necessary networking facilities to optimise the use of network resources.

After deployed the selection of sessions to be further established towards a user (or set of them), it is necessary to derive the quality level by selecting a proper codec. Afterwards, it is necessary to allow the propagation of the session along a communication path. Firstly, it is necessary to derive the characteristics of the session, in terms of connectivity (unicast or multicast) and QoS (e.g., peak rate and maximum tolerance to delay/loss). In order to optimize complexity of such operations in heterogeneous environments, a generalized transport scheme can be designed to allow a common handling of the session by all networks between the source and the receivers, independent of the underlying technologies. Moreover, it will enable each network to choose their own schemes and mechanisms to operate in the control plane to setup network resources. To keep users best connected over the time, it is necessary to select the best network interface to activate the session content reception.

\section{CONTEXT CASTING INITIAL ARCHITECTURE}

In order to provide personalized services to mobile users in the future Internet, three frameworks are designed in a C-CAST enabled network. Context sensed in mobile terminals and collected from network devices is deployed in the Context Acquisition and Delivering framework. The selection of content sessions, based on user context, is done in the Context to Content Selection framework. Finally, the resource configuration and session quality control is performed in the Session Establishment and Management framework.

\section{A. Context Acquisition and Delivering Framework}

The components in the Context Acquisition and Delivering framework aim to detect context about mobile terminals and 
network devices to make available for Context Consumer. The user context is collected by sensors (attached or not to terminals), dealing with information about the physical environment of the users, as well as terminal capabilities, availability and status. The user context is sensed taking into account resolution, accuracy and period of time requested by the context consumer. The list of user context can include: location and proximity (RFID (indoor) and GPS (outdoor)); cell ID; supported network interfaces (multihomed); capabilities of each network interface; connectivity support (multicast); battery status; ambient information (temperature, luminance, relative humidity). Fig. 1 shows the components of the Context Acquisition and Delivering framework and interactions with other components.

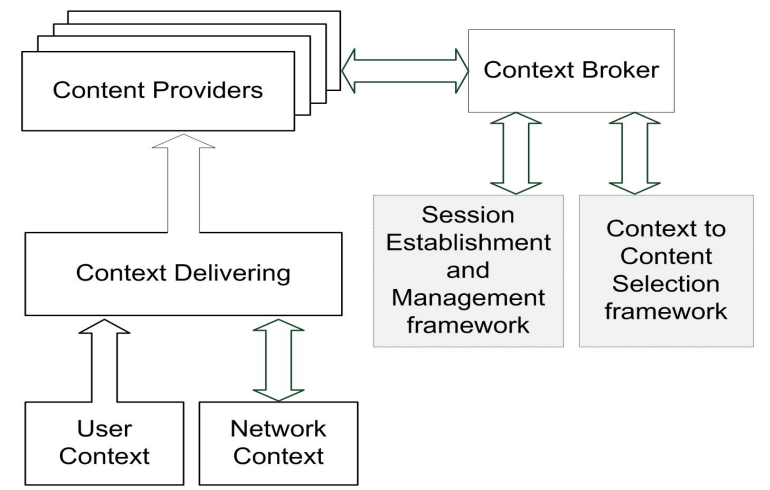

Fig. 1. Main components in the Context Acquisition and Delivering framework in a C-CAST enabled network.

In what concerns network context, the goal is to collect information anyway available today in MIB of network devices (e.g., modems, base stations and routers). Hence, it is possible to collect information about traffic statistics (loss ratio, packet load); QoS information (available classes), packet forwarding (local multicast trees), network capabilities (IP address of interfaces, capacity, technology), routing and etc. Session context (QoS requirements, list of destinations) and environmental context (location, velocity, noise, and presence) can also be used in the overall framework. A well-defined API will be used to retrieve context of user, session, network and environment. After sensed, the Context Delivering must supply a Context Provider with the collected context. A set of Context Providers can be designed to provide context from different perspectives. The interface between the Context Providers and the Context Consumers is the Context Broker.

\section{B. Context to Content Selection}

The selection of content sessions and destination users is deployed in the Context to Content Selection framework. The main component is called Context to Content Application, which uses pre-defined rules for reasoning in user context to match potential content sessions. Optionally to on-demand/periodic requests, the Context Broker can be configured to send context to the Context to Content Application asynchronously. For instance, the Context Broker can be configured to send context of new users attached to a certain cell or located around a certain area. Thus, a set of users can be advertised with indications in the case of catastrophes rounding their location.

The Context to Content Application matches content sessions that are provided by a set of well-known Content Sources, which can be advertised by existing mechanisms, such as SIP [9]. The Group Management is responsible to compose groups of users that will receive the selected content session(s). Fig. 2 shows the components, and how they interact, of the Context to Content Selection framework.

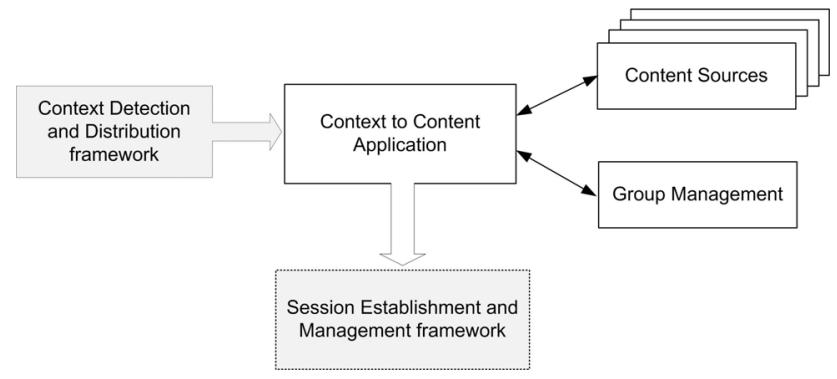

Fig. 2. Components of the Context to Content Selection framework

\section{Session Establishment and Management}

After matching potential content sessions and composed groups of users, the sessions and network resources can be provided. The main objective of the components of the Session Establishment and Management framework is to provision session requirements and network resources to allow the efficient support of multi-party sessions over the time to one or many destination users. Fig. 3 depicts the components of the Session Establishment and Management framework and their interaction via a well-defined API, the Session Management (SM), the Network Use Management (NUM), the IP Transport (IPT) and the Mobility Controller (MC).

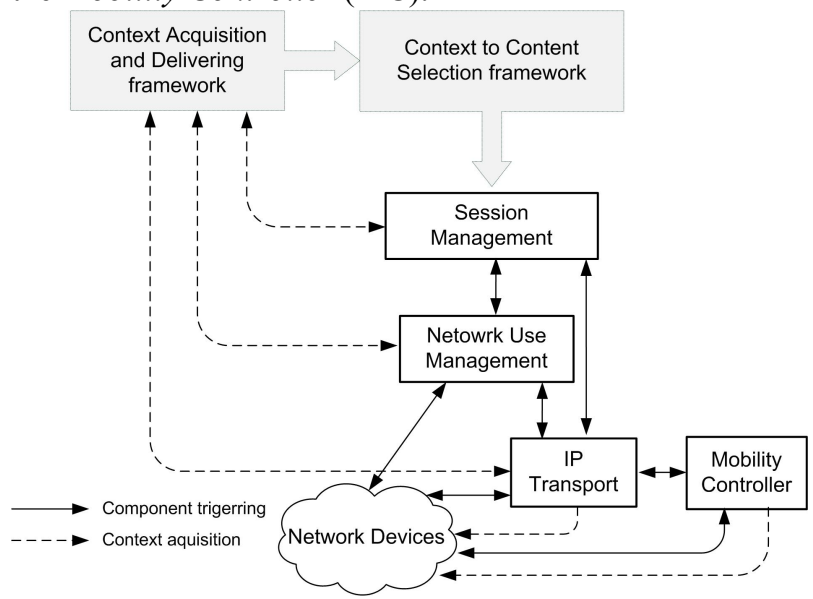

Fig. 3. Components of the Session Establishment and Management framework in a C-CAST enabled network.

The general requirements of the Session Establishment and Management framework include: Keeping multihomed mobile terminals always best connected; Allowing mobile terminals to receive content sessions even during re-routing events; Allowing the dynamic setup and re-negotiation of sessions according to context information; Supporting scalable schemes for resource allocation and signalling to coordinate the dynamic setup of network elements; Supporting IP multicast to control the creation 
of distribution trees in asymmetric environments; Supporting scalable and fast operations for resilience; Collecting user, session, network and environment context. The operations and functionalities supported by each component of the Session Establishment and Management framework are described in the following section.

\section{Session Establishment AND MANAGEMENT}

This framework enables the support of dynamic multiparty sessions, with changing conditions depending on the context, and implements an abstract transport scheme to provide best access to the users for its sessions. Moreover, it allows constant network changes due to context with minimal impact to the abstract transport trees.

\section{A. Session management}

The Session Management (SM) is the functional entity in charge of efficiently establishing communication sessions to propagate flows to groups of users with QoS requirements. Such efficiency deals with adapting the requirements of the session content to the access network capabilities over the lifetime. The QoS requirements of the session are derived by SM based on network conditions/capabilities (available resources, supported technologies, traffic conditions), content characteristics (codec info, e.g., bit rate) and user capabilities (available network technologies, velocity, noise measures, light amplitude). User and network context are retrieved from the Context Acquisition and Delivering framework.

The Session management should be triggered by the Context to Content Application requesting the establishment of a multiparty session for a group of users demanding the same information. Based on that, the SM should request the most appropriated content that better fits the group of user context (needs and preferences). The SM needs to know the access network to be used by the terminals as well as network context, which is done by interacting with the Network Use Management (NUM). Moreover, SM must select an appropriate quality level required to efficiently propagate a content session. Such operation encompasses correctly assigning class of service, maximum delay/loss ratio, peak bit-rate, and so on. The SM can also modify previous QoS requirements of the session to perform adaptation, which is necessary under re-routing or any other context condition.

There are three types of interaction between SM and NUM. In the first one, SM explicitly requests network context in terms of QoS capabilities supported in the selected communication path associated with the content session intending to establish. In the second one, NUM informs SM about the need to modify the session that is running for a specific user or group of users. This situation occurs whenever a change in the network is necessary (congestion or handover detection). In this case, NUM notifies SM with the network to be used and the available QoS description. In the third case, the SM notifies the NUM when changes in the session were in place due to change in context, to adapt the network to the new session conditions. This can happen, for example, when audio is removed from a session due to the large noise in the environment and inability to ear the audio session.

\section{B. Network Use Management}

NUM has the capability to provide intelligent context-aware network selection. Therefore, a multimode terminal is expected to always receive multiparty sessions using the most appropriated network interface, with multihomed terminals receiving simultaneously flows of multiparty content sessions in different interfaces (they may even switch between interfaces to prevent quality degradation or according to changes in the context), and switch between unicast and multicast modes according to the context information received and the network support. Current attempts with limitations in the support of end-host mobility with multihoming are the Stream Control Transmission Protocol (SCTP) [10] and the Host Identity Protocol (HIP) [11] mechanisms. On one hand, SCTP has no support to simultaneous communication on different interfaces, reordering and load balancing, as well as it is not able to provide information on how to select the best path. On another hand, HIP evolves towards mobility management without support to provide information about technologies and QoS attributes of local network interface.

Based on the limitations of existing proposals, a new scheme is required to efficiently support network selection based on different inputs (network, user, session and environment context). The heterogeneity aspect of future Internet requires routing decisions to take into account the underlying transport capabilities, but also all the relevant information of the users and sessions, as well as the environmental information, such as location and velocity.

The context information list required by NUM may contain the following (but not limited to):

- Network context: available communication paths, available classes; minimum reserved/used bandwidth; traffic statistics; Access Points in range and their capabilities; network technology; connectivity support (multicast/unicast);

- User and terminal context: network interfaces: ID, technologies, capabilities, current network usage; signal strength; contracts; preferences; connectivity support;

- Session context: QoS requirements (class, peak bit rate, maximum loss/delay tolerance); destinations;

- Environmental context: location, velocity, noise, presence.

Since we envision a very dynamic environment with dynamic context influencing session and network control, we consider the use of abstract transport to allow an abstract transport control in the multiparty trees to hide the dynamicity of the network and session changes in the multiparty session and tree. The main idea resides in creating an abstract data path which is composed by the several sub transport structures, for further merging with multiple incoming multiparty content sessions. Abstract transport allows an end-to-end view in terms of connectivity (Abstracted Multiparty Tree - AMT), which can be composed by several Sub- 
AMT. Thus, each network between the Content Source and destination users can control network resources with solutions and technologies of their choice, with minimal impact in the abstract trees. The NUM is the element that controls the abstract trees. The Sub-AMT must be mapped to communication paths with allocated network resources, to guarantee a QoS-enabled transport. To do that, NUM must also interact with the IP transport component described in the next section. When some context changes and it is required to change network configurations and internal routing, NUM is in charge to control it with the IP Transport. The AMTs are only notified if changes are required to them. If not, they are seamless to the changes, and therefore, the sessions, senders and receivers will also be transparent to these changes.

\section{IP Transport}

The IP Transport (IPT) has as main goal to allow the dynamic allocation of network resources for the efficient propagation of multi-party content sessions along QoS-aware IP multicast trees. The technical requirements of IPT include:

- Support adequate principles and functions to setup intra- and inter-domain network resources;

- Negotiate QoS level, taking into account user, network session and environmental context;

- Support centralized and/or distributed operations;

- Detect topology changes and deploy efficient operations for reliance;

- Interact with NUM to support the changes in network without affecting the AMTs;

- Notify the AMTs of changes requiring AMTs or sub-AMTs;

- Support new concepts/functionalities to deploy scalable and efficient allocation of network resources;

- Control the population of the Multicast Routing Information Base coupled with resource allocation functions to provide QoSaware IP multicast trees, as well as allocate IP multicast addresses;

- Interact with mobility controllers to detect handovers in order to re-establish the affected session in another path;

- Support different mobility technologies, in order to allocate network resources in wireless networks.

IPT collects Context information from network devices or from the components of the Context Acquiring and Delivering framework, via a well-defined API. IPT must be aware about local technologies and network elements (e.g., QoS model, packet scheduling and IP multicast protocol) to efficiently deploy resource allocation and build IP multicast trees (when applicable). Interactions with the SM are required to determine the QoS required by the multi-party content session. Additionally, SM can be triggered for resilience, where for instance adaptation and/or re-mapping can be deployed to adjust multi-party content session quality to the current network conditions.

Due to the performance limitations of current QoS models and resource allocation solutions, IPT will take as basis solutions based on the coupled control of resource reservations and multicast tree creation ([12][13]). These mechanisms need then to be integrated with NUM and AMT to allow the support of abstract multicast trees on top with intelligent and optimized selection of networks and connections. The context information list required by IPT may contain at least: (i) Network Context: available communication paths; edge-to-edge QoS bottleneck capabilities of each communication path; (ii) Terminal context: location, IP address; (iii) Session context: QoS requirements, destination users; (vi) Environmental context: velocity, presence.

\section{Mobility Controller}

Mobility is, from one side, the result of the context changes that require the change of points of access for the several multiparty users and sessions and, from the other side, one of the context sources that will require changes in both sessions and networks. For these reasons, the mobility controller interacts, indirectly with NUM and Context Acquisition, in the first case for the NUM to request the terminal to move according to its decision, and in the second case, to use the mobility as a source of context that will require both changes in the session and network.

One important requirement for mobility is that the network is able to re-configure without impact on the running sessions. The use of AMTs allow the support of trees based on the sessions and abstracted from the network, which will support the constant changes in the network due to mobility without impacting on the AMTs, producing mobility- and session-aware trees. A tight coupling between NUM and IPT is required for this support. In a future all-mobile environment, with both sources and receivers being mobile, where any terminal can provide content to other users, new mobility models need to be devised to decouple the senders from the receivers mobility and minimize the impact in each node's mobility. Again, the support of new mobility- and session-aware trees together with sources and receivers decoupling mobility will be crucial for the support of future Internet.

\section{Practical Use CASE}

This section presents a practical use case, and how the envisioned architecture may cope with such dynamic scenario.

"The F1 Grand Prix is back to town, and fans all over the world travel to Estoril to support their favorite pilots. While on move, groups of friends are watching the F1 channel. While Alonso supporters are receiving the videos from Renault cars, Hamilton fans are getting information about the new McLaren wheels. During the trip they are always switching between different networks, but the video is continuously playing, always matching the terminal capabilities.

Today is the big day. Because the tickets machine were giving context information to the network informing the number of tickets sell, all the networks have been automatically reconfigured in order to better accommodate such a huge demand of communications. In the mobile screens, users are receiving images from their favorite pilots or their favorite turns. When the 
cars start putting the engines on, the network decreases all the audio quality because it is imperceptible for end users. Furthermore, due to the movement sensors, the network becomes aware about users that are not focus on the mobile and, consequently, may switch off their connections. Because most of the people like to replay the car accidents, whenever there is a car crash, the video retransmits it and adds a set of related information, such as velocity and so on. During the competition, personalized advertisements are sent directly to groups of users."

Before reaching a wireless access network, it is required to establish the multi-party content session along a core network, or a set of them. In order to setup a multi-party content session, NUM must be triggered by the SM placed at the first core network indicating the session context and the destination user(s). First of all, NUM must retrieve user and network context in the Context Acquisition and Delivering framework. NUM can use the terminal information to retrieve (e.g., via BGP) the local egress router. Then, NUM is allowed to collect network context, which can be done by probing the traffic statistics and available resources along the path towards the egress router. Alternatively, a Context Provider can be designed to collect network context and further make it available. Based on the session and network context, NUM is able to assign network requirements for the traffic. Afterwards, NUM creates an Abstracted Multiparty Tree (AMT), and one sub-AMT, and triggers IPT to setup network resources. IPT allocates an IP multicast address and signals the communication path. Each visited node sets up the resource reservation and the IP multicast tree. After finished the session setup, NUM provides the SM with the IP addresses. SM triggers (via SIP) each mobile terminal of the group to join the multiparty content session.

Beyond this normal behavior for setting up sessions to groups of users in the best networks, several changes of context need to be undertaken. For example, in handovers during the trip, the mobility of users is triggering the IPT, and then the NUM, to decide the best networks to connect to based on context, including location. If changes are required in the network, NUM will contact IPT to setup resources in the new network, while it constructs new sub-AMT only if the previous ones do not accommodate the session changes. If changes are required in the sessions to accommodate the new network conditions, NUM will contact SM for session re-negotiation. In the situation of context information by the ticket machines, the NUM is contacted by the Context Broker and determines the best networks to attach current users to give space for the new demand of communications. This change will require new abstract trees for the change of the ongoing sessions, and consequent IPT updating the network resources. The Mobility controller is also triggered to move the ongoing sessions to the optimal points of access. Moreover, this movement may require the sessions to renegotiate their parameters, and the SM is contacted for this purpose. In the situation of large noise due to cars engines, SM can react (due to Context Broker triggering) to adapt the session (removing audio). This will also have impact on the network part, and IPT is invoked to update the network resources. If this context change requires new optimizations in the network, NUM will determine the best connections for the multiparty sessions, and the decisions are mapped in IPT.

\section{CONCLUSION}

It is evident now that, to add a new flavour to the network convergence, a close coupling between creation of context awareness and mobile multicasting technologies is necessary to support end-to-end context-aware real-time communication, specifically for intelligent personalized, user specific and dynamic multicast-broadcast services. This paper provided key points and requirements to ensure a successful evolution of mobile communication networks towards futuristic Ambient Intelligence environment / ubiquitous smart spaces / pervasive environment, where different multiparty communication behaviours, such as user-initiated communication mediated by the network and situation-oriented network-initiated communication mediated by the environment, will become possible. We believe that this will stimulate new research efforts to address the overwhelming needs for future Internet technologies. As future work, we plan to devise the algorithms for the session establishment and management framework, namely the algorithms for intelligent network selection and construction of abstract multiparty trees in dynamic context-aware environments, and assess its scalability when compared to the mechanisms support in current networks.

\section{REFERENCES}

[1] FP7 ICT-2007-216462, Context Casting, Funding, EU-FP7 ICT

[2] R. Ocampo, L. Cheng, A. Galis, "ContextWare support for network and service compositions and self-adaptation", Proc 2nd Int'l Work. Mobility Aware Tech. \& Application, 2005.

[3] H. Chen, T. Finin, A. Joshi, "An intelligent broker for context-aware pervasive computing environments", Proc. Ubicomp, 2003.

[4] S.S. Yau, F. Karim, "A lightweight middleware protocol for ad hoc distributed object computing in ubiquitous computing environments", Proc. 6th IEEE Int'1 Symp. Object-Oriented Real-Time Distributed Computing, 2003.

[5] B. Sigrid, M. Poi, U. Tore, U, "A simple architecture for delivering context information to mobile users", Proc. Work. Infrastructure for Smart Devices - How to Make Ubiquity an Actuality, 2003.

[6] SMART-ITS (IST-2000-25428) Interconnected Embedded Technology for Smart Artefacts with Collective Awareness.

[7] WORKSPACE (IST-2000-25290) Distributed WORK support through component based SPAtial Computing Environments.

[8] GLOSS (IST-2000-26070) Global Smart Spaces.

[9] J. Rosenberg, G. Camarillo, "SIP: Session Initiation Protocol", RFC 3261 June 2002.

[10] R. Stewart et al., "Stream Control Transmission Protocol (SCTP) Dynamic Address Reconfiguration”, Internet Draft draft-ietf-tsvwg-addip-sctp-06.txt, work in progress, IETF, September 2002.

[11] R. Moskowitz, "Host Identity Protocol" IETF Internet Draft (Work in Progress), July 2006.

[12] A. Neto, E. Cerqueira, A. Rissato, E. Monteiro, and P. Mendes, "A Resource Reservation Protocol Supporting QoS-aware Multicast Trees or Next Generation Networks", In Proc. 12th IEEE Symp. on Computers and Communications, Aveiro, Portugal, July 2007.

[13] E. Guainella et al, "QoS Management of Multicast and Broadcast Services in Next Generation Networks", 16th IST Mobile \& Wireless Communications Summit, July 2007. 